\title{
PTU-022 FAECAL CALPROTECTIN AS PART OF STANDARDISED ASSESSMENT TOOL OF SUSPECTED IRRITABLE BOWEL SYNDROME (IBS): A NOVEL INVESTIGATIVE ALGORITHM
}

doi:10.1136/gut.2011.239301.150

A Ramadas, ${ }^{*}$ K Datta, L Sunderraj, ${ }^{1}$ A B Hawthorne ${ }^{1}$ Department of Gastroenterology, University Hospital of Wales, Cardiff, UK

Introduction There is enormous variability in the referral rates for IBS from GPs and this relates to patient and physician factors. ${ }^{1}$ IBS patients tend to wait longer for appointments and hence a standardised algorithm which includes faecal calprotectin (FC) might aid in the rapid assessment of IBS in secondary care. FC has been shown to consistently differentiate IBS from IBD because it has excellent negative predictive value in excluding organic pathology. ${ }^{2}$

Methods To assess the efficacy of diagnostic algorithm (using FC) for assessment of IBS in a pilot study using patients already referred to hospital GI clinics. Patients were included if the GP suspected IBS (age group 18-50),without alarm symptoms (weight loss, rectal bleeding etc) at time of referral. Initial assessment was in a nurse-led clinic using a standardised algorithm consisting of questionnaire and baseline investigations (FC, FBC, ESR, CRP, albumin, IgA and TTG). FC level < 100 $\mathrm{mcg} / \mathrm{g}$ was considered normal. ${ }^{3} 4$ week review was undertaken by a doctor and those with abnormal results were transferred to the GI clinic. 1-year assessment was undertaken on all study patients, reviewing final diagnosis and hospital visits. Clinical diagnosis by nurse and doctor before results of FC and blood tests were compared. Co-primary end-points were the positive predictive value (PPV) of the algorithm in diagnosing IBS and the added benefit of FC in excluding organic pathology. 
Results 54 patients were recruited and 51 attended the 4 -week follow up. 49/54 (90\%) had complete 1-year follow-up data. Reason for GP referral was 'uncertain diagnosis' in 37/54 (69\%) and 'confirmation of IBS' in 17/54 (31\%) patients. There was $95 \%$ concordance between nurse and doctor in the clinical diagnosis of IBS (blinded to blood results and FC) based on Rome criteria. 44/54 (81\%) were diagnosed as IBS at 4-week review and none had evidence of organic pathology at 1 year. Non-IBS diagnosis 5/54 (9\%) included 3 IBD and 2 Coeliacs. Median FC levels in the IBS and non-IBS groups were 19.5 and 1105 respectively $(p=0.0001)$. Two patients in the IBS group were re-referred within 1-year (final diagnosis IBS). PPV of the algorithm in diagnosing IBS was $100 \%$ without FC, but FC clearly identified the IBD patients and a normal level $(96 \%$-ve predictive value) provided valuable reassurance.

Conclusion This diagnostic algorithm is useful for a nurse-led clinic for assessment of suspected IBS referred to hospital, and FC measurements improve the diagnostic confidence of nurses and doctors, and removes the need for endoscopic investigation in patients under 50 .

Competing interests None.

Keywords irritable bowel syndrome, faecal calprotectin.

\section{REFERENCES}

1. Spiller et al. Gut 2007:56:1770-98.

2. Van Rheenen et al. BMJ 2010;341:C3369.

3. Dolwani et al. Aliment Pharmacol Ther 2004;20:615-21. 